Article

\title{
Origin and Distribution of the $V R N-A 1$ Exon 4 and Exon 7 Haplotypes in Domesticated Wheat Species
}

\author{
Alexandr Muterko* (D) and Elena Salina \\ The Federal Research Center Institute of Cytology and Genetics, Lavrentyeva Avenue 10, \\ Novosibirsk 630090, Russia; salina@bionet.nsc.ru \\ * Correspondence: muterko@bionet.nsc.ru; Tel.: +7-383-363-49-63 (ext. 3211)
}

Received: 5 July 2018; Accepted: 17 August 2018; Published: 20 August 2018

\begin{abstract}
The high adaptive potential of modern wheat to a wide range of environmental conditions is determined by genetic changes during domestication. Genetic diversity in VRN1 genes is a key contributor to this adaptability. Previously, the association between the transitions $C->\mathrm{T}$ within the fourth and seventh exons of $V R N-A 1$, the distinguishing pair haplotypes Ex4C/Ex4T and Ex7C/Ex7T, and the modulation of such agronomically valuable traits as the vernalization requirement duration, frost tolerance and flowering time of wheat have been shown. However, this polymorphism was analyzed in only a few cultivars of Triticum aestivum $L$., and not in other wheat species. In the present study, $V R N-A 1$ exon 4 and exon 7 were investigated in six tetraploid and five hexaploid wheat species carrying different $V R N-A 1$ alleles. An allele-specific polymerase chain reaction (PCR) assay was optimized to identify the $V R N-A 1$ exon 7 haplotypes. It was found that polymorphism of the $V R N-A 1$ exon 7 originated in wild tetraploid wheat of Triticum dicoccoides Körn, while the mutant exon 4 of this gene originated later in domesticated hexaploid wheat of T. aestivum. Both these polymorphisms are found in all hexaploid wheat species. Analysis of the $V R N-A 1$ exon 4 and exon 7 haplotype combinations found that intact exon 7 and mutant exon 4 are associated with analogous types of exon 4 and 7, respectively. With the exclusion of the Vrn-A1c (IL369) and Vrn-A1j alleles, identified only in hexaploid wheat, all dominant $V R N-A 1$ alleles carry intact exons 4 and 7 (Ex4C/7C haplotype). The Ex4C/4T/7T haplotype was detected in numerous accessions of hexaploid wheat and is associated with the presence of multiple copies of $V R N-A 1$. Overall, modern domesticated hexaploid wheat T. aestivum includes most possible combinations of the $V R N-A 1$ exon 4 and exon 7 haplotypes among polyploid wheat, which are present in different proportions. This contributes to the high adaptive potential to a broad range of environmental conditions and facilitates the widespread distribution of this species throughout the world.
\end{abstract}

Keywords: wheat; adaptability; genetic diversity; VRN-A1 exon 4; exon 7; polymorphism; haplotypes

\section{Introduction}

Wheat was one of the first crops to be domesticated and now comprises a key source of raw material for the food and processing industry. According to the prevailing view, hulled emmer Triticum dicoccum Schrank (BBAA genomes) was domesticated approximately 10,000 years ago from wild emmer T. dicoccoides. From domestic emmer, in turn, arose the free-threshing tetraploid wheat (T. durum) [1]. Hexaploid wheat (T. aestivum, BBAADD genomes) was then derived from the hybridization of tetraploid wheat with the goat grass Aegilops tauschii Coss. (DD genome) [2].

Following the domestication of wheat, the cultivation of this crop began to spread widely beyond the confines of the fertile crescent [1]. The culmination of this trend means that currently more of 
the earth's surface is covered by wheat than by any other food crop [3]. It's distribution throughout very different eco-geographical areas demands the high adaptability of wheat to a wide range of environmental conditions. This adaptability, which enables wheat to time its flowering to occur when climatic conditions are most favorable, is mostly determined by allelic diversity within genes controlling the seasonal growth habit (vernalization requirement) and photoperiod sensitivity [4-6].

The need for prolonged exposure to low temperatures in order for plants to become capable of flowering is determined by the vernalization requirement. Vernalization sensitivity is an important trait of wheat, determining the flowering time and yield through adaptability to the environment [6]. This is especially important in wheat breeding in conditions of changing climates. The vernalization response in wheat is largely controlled by the four VERNALIZATION genes: VRN1 (a MADS-box transcription factor), VRN2 (a zinc-finger CCT domain gene, ZCCT), VRN3 (homologous to the Arabidopsis gene FLOWERING LOCUS T) and VRN-D4 (a MADS-box transcription factor, derived from the duplication of the $V R N-A 1$ gene in the short arm of chromosome 5D) [7-10]. However, the features of the allelic distribution of the different $V R N$ genes and their effect on the phenotype make VRN1 the key determinant of the vernalization requirement in polyploid wheat [11].

The dominant VRN1 alleles, which determine a spring growth habit, carry mutations within the promoter region or in the first intron ([11,12], and references therein). Among the three homologous VRN1 genes, polymorphism in the coding sequence of the recessive allele (determining a winter growth habit), which is associated with the modulation of such agronomically valuable traits as the frost tolerance, vernalization requirement duration, days to stem elongation and flowering time of wheat, has been revealed only for the VRN-A1 gene [13-16].

Depending on the nucleotide at position $20 \mathrm{bp}$ of the VRN-A1 fourth exon, the $\mathrm{C}(\mathrm{Ex} 4 \mathrm{C}$, wild type) and $\mathrm{T}$ (Ex4T, mutant type) haplotypes are distinguished [13]. Missense mutation caused by the "C->T" transition within exon $4 V R N$ - $A 1$ leads to the substitution of conservative leucine for phenylalanine $\left(\mathrm{Leu}^{117}->\mathrm{Phe}{ }^{117}\right)$ in the amino acid sequence of the K-domain. In previous studies, this polymorphism was associated with a change in the number of days to stem elongation, vernalization requirement duration, frost tolerance and flowering time of the winter wheat of T. aestivum [13,14].

The other important single nucleotide polymorphism (SNP) within the VRN-A1 coding sequence is the "C-> T" transition in the exon 7 , resulting in the substitution of alanine for valine ( $\mathrm{Ala}^{180} / \mathrm{Val}^{180}$ ) in the amino acid sequence [17]. Despite the localization of this mutation in the C-terminal of the $V R N-A 1$ protein, where no functional domains have been identified, the polymorphism in this site is associated with a change in the vernalization requirement duration and flowering time of wheat [15]. Furthermore, it was reliably established that the missense mutation $\mathrm{Ala}^{180} / \mathrm{Val}^{180}$ in VRN-A1 influences the interaction of this protein with the expression products of TaHOX1, which affects the flowering time [15].

In hexaploid wheat, the $V R N-A 1$ gene can be present as two or more copies per haploid genome $[18,19]$. There is an assumption that the number of $V R N-A 1$ copies positively correlates with the vernalization requirement duration and flowering time of wheat [18]. However, since different copies of $V R N-A 1$ contain polymorphisms within exon 4 and exon 7 , which, as was shown later, affects the same traits, this assumption is debatable [15].

In our recent study, analysis of the distribution of the $V R N-A 1$ exon 4 haplotypes in 12 tetraploid and hexaploid wheat species and in different $V R N-A 1$ alleles, found the mutant type of exon 4 (Ex4T haplotype) only in hexaploid wheat with a recessive vrn- $A 1$ allele, and only in combination with the intact exon 4 (Ex4C haplotype) of this gene (i.e., in accessions of hexaploid wheat carrying no less than two copies of $V R N-A 1$ per genome) [20]. However, the polymorphism of the $V R N-A 1$ exon 7 was previously analyzed only in some varieties of $T$. aestivum, but not other polyploid wheat species. Often, exons 4 and 7 of $V R N-A 1$ were analyzed separately. In most cases, analysis was carried out without taking into account the copy number variation of the $V R N-A 1$ gene, which is especially important for hexaploid wheat, where, for example, in T. aestivum, more than $90 \%$ of winter varieties carry 2-3 copies of this gene [19], with different combinations of the exon 4 and exon 7 
haplotypes $[15,16,18]$. Furthermore, there are only four SNPs between the VRN-D4 and VRN-A1 DNA sequences [10]. Hence, the results of previous studies should also be reviewed, taking this into account, since using existing methods of analysis, it is not possible to distinguish exons 4 and 7 of $V R N-A 1$ and those of $V R N-D 4$. This problem was resolved in our recent study through design of allele-specific primers and the optimization of PCR for the identification of $V R N-D 4$ and distinguishing between $V R N-A 1$ and $V R N-D 4$ haplotypes. Furthermore, the co-dominant DNA markers, based on the modulation of the curvature of the DNA molecule were designed to enable discrimination between the $V R N-A 1$ exon 4 haplotypes [20].

In the present study, using an optimized allele-specific polymerase chain reaction (PCR) assay, we analyze polymorphism in the 7th exon of $V R N-A 1$ in 11 species of polyploid wheat carrying different $V R N-A 1$ alleles. Furthermore, we identify the different combinations of the $V R N-A 1$ exon 4 and exon 7 haplotypes to shed light on their origin, divergence and distribution in modern domesticated wheat species. Finally, we use this information for the reconstruction of the important evolutionary events in wheat evolution, such as copy number variation and divergence of $V R N-A 1$ alleles. Taken together, these data allow us to evaluate how genetic changes during domestication have affected agronomically valuable traits of modern domesticated wheat species, broadening their adaptive potential to a wide range of environmental conditions.

\section{Materials and Methods}

\subsection{Plant Material}

A total of 190 accessions representing 5 hexaploid and 6 tetraploid wheat species from different eco-geographic areas were investigated. The hexaploid species (genomes $B B A^{u} A^{u} D D$ ) included were T. aestivum L., T. spelta L., T. macha Dekap, T. compactum Host, T. sphaerococcum Percival. The tetraploid species (genomes $B B A^{u} A^{u}$ ) consisted of T. durum Desf., T. turgidum L., T. polonicum L., T. carthlicum Nevski, T. dicoccum Schrank and T. dicoccoides Körn. Germplasm was obtained from the National Plant Germplasm System (NPGS, Washington, DC, USA), Leibniz Institute of Plant Genetics and Crop Plant Research (IPK Gatersleben, OT Gatersleben, Stadt Seeland, Germany, National Centre for Plant Genetic Resources of Ukraine (Kharkiv, Ukraine), International Center for Agricultural Research in the Dry Areas (ICARDA, Beirut, Lebanon) and Vavilov Institute of Plant Genetic Resources (VIR, Saint Petersburg, Russia).

\subsection{Nucleic Acid Extraction and Amplification}

Total nucleic acid (DNA and RNA) from 3-day-old wheat seedlings was extracted following a slightly modified CTAB method [21].

Polymerase chain reaction (PCR), in a volume of 3-6 $\mu \mathrm{L}$, consisted of: DNA (100 ng), $20 \mathrm{mM}$ Tris, $10 \mathrm{mM}\left(\mathrm{NH}_{2}\right)_{2} \mathrm{SO}_{4}, 2.1-2.6 \mathrm{mM} \mathrm{MgCl}_{2}, 1 \mathrm{mM} \mathrm{KCl}, 0.1 \%$ Triton X-100, $234 \mu \mathrm{M}$ each dNTP, $1.5 \%$ dimethyl sulfoxide (DMSO), $4 \mathrm{ng} / \mu \mathrm{L}(0.5 \mu \mathrm{M})$ each primer and $0.05 \mathrm{U} / \mu \mathrm{L}$ Taq-polymerase. Mineral oil $(7.5 \mu \mathrm{L})$ was overlaid on top of the reaction mixture to prevent evaporation. The PCR was performed using the following program: denaturation at $96^{\circ} \mathrm{C}(3 \mathrm{~min}) ; 33$ cycles of amplification: $93^{\circ} \mathrm{C}(10 \mathrm{~s})$, annealing (10 s), $69^{\circ} \mathrm{C}(50-90 \mathrm{~s})$ per cycle, and a final elongation step of $69^{\circ} \mathrm{C}$ for $3 \mathrm{~min}$. Further details of all primers, including annealing temperatures, are listed in Table 1. 
Table 1. PCR primer details.

\begin{tabular}{|c|c|c|c|c|c|c|}
\hline Primer & Sequence $\left(5^{\prime}-3^{\prime}\right)$ & $\begin{array}{l}\text { Primer } \\
\text { Developer }\end{array}$ & $\begin{array}{c}\text { Annealing } \\
\text { Temperature }\left({ }^{\circ} \mathrm{C}\right)\end{array}$ & Region & $\begin{array}{l}\text { Allele or } \\
\text { Haplotype }\end{array}$ & $\begin{array}{c}\text { PCR } \\
\text { Fragment } \\
\text { Size (bp) }\end{array}$ \\
\hline VRN1AF & gaaaggaaaaattctgctcg & \multirow{2}{*}{ [22] } & \multirow{2}{*}{58} & \multirow{2}{*}{$\begin{array}{l}\text { VRN-A1 } \\
\text { promoter }\end{array}$} & vrn-A1 & 713 \\
\hline \multirow[t]{14}{*}{ VRN1-INT1R } & gcaggaaatcgaaatcgaag & & & & $V r n-A 1 a .1$ & 944 \\
\hline & & & & & $V r n-A 1 a .2$ & 924,944 \\
\hline & & & & & Vrn-A1a.3 & 765 \\
\hline & & & & & $V r n-A 1 b$ & 691 \\
\hline & & & & & $V r n-A 1 d$ & 685 \\
\hline & & & & & Vrn-A1e & 659 \\
\hline & & & & & Vrn-A1f & 658 \\
\hline & & & & & $V r n-A 1 i$ & 713 \\
\hline & & & & & $V r n-A 1 j$ & 659,713 \\
\hline & & & & & Vrn-A1k & 755 \\
\hline & & & & & vrn- $A^{m} 1$ & 705 \\
\hline & & & & & $V r n-A^{m} 1 g$ & 681 \\
\hline & & & & & $V r n-A^{m} 1 a$ & 671 \\
\hline & & & & & vrn- $A^{m} 1 b$ & 656 \\
\hline Vrn-A1-intr_F & ccgtcgaaaggatcgctactg & \multirow{2}{*}{ [12] } & \multirow{2}{*}{60} & \multirow{2}{*}{$\begin{array}{c}V R N-A 1 \\
\text { intact intron-1 }\end{array}$} & vrn-A1 & 541 \\
\hline Vrn-A1-intr_R1 & cttgtccccgtgagctacttac & & & & & \\
\hline $\mathrm{Ex} 1 / \mathrm{C} / \mathrm{F}$ & gttctccaccgagtcatggt & \multirow[t]{2}{*}{ [23] } & \multirow[t]{2}{*}{56} & \multirow{2}{*}{$\begin{array}{c}V R N-A 1 \\
\text { intron-1 with } \\
\text { large deletion }\end{array}$} & $\begin{array}{c}\text { Vrn-A1c } \\
\text { (Langdon) }\end{array}$ & 522 \\
\hline Intr1/A/R3 & aagtaagacaacacgaatgtgaga & & & & Vrn-A1c (IL369) & 2188 \\
\hline Ex4F2m3 & ttgttccttcctgtcccaacc & \multirow{2}{*}{$\begin{array}{l}\text { [14] (modified } \\
\text { by [20]) }\end{array}$} & \multirow{2}{*}{60} & \multirow{2}{*}{$\begin{array}{c}V R N-A 1 / V R N-D 4 \\
\text { exon-3-4 }\end{array}$} & $\operatorname{Ex} 4(\mathrm{C} / \mathrm{T})$ & 389 \\
\hline Ex4R & ctttgctgaacttctctgc & & & & Ex4C.f & 387 \\
\hline Ex4F2m3 & ttgttccttcctgtcccaacc & \multirow{2}{*}{ [20] } & \multirow{2}{*}{59} & \multirow{2}{*}{$\begin{array}{l}V R N-D 4 \\
\text { exon-3-4 }\end{array}$} & VRN-D4 & 265 \\
\hline $\mathrm{V} 4 / \mathrm{R} 2 \mathrm{~m} 3$ & ccagttgctgcaactccagg & & & & & \\
\hline Ex4outV4F2m3 & cctgtcccacccaaagttacta & \multirow{2}{*}{ [20] } & \multirow{2}{*}{57} & \multirow{2}{*}{$\begin{array}{l}V R N-A 1 \\
\text { exon-3-4 }\end{array}$} & $\operatorname{Ex} 4(\mathrm{C} / \mathrm{T})$ & 380 \\
\hline Ex4R & ctttgctgaacttctctgc & & & & Ex4C.f & 378 \\
\hline $\mathrm{Ex} 4 \mathrm{~F} 2 \mathrm{~m} 3$ & ttgttccttcctgtcccaacc & \multirow{4}{*}{ [20] } & \multirow{4}{*}{58} & \multirow{4}{*}{$\begin{array}{l}V R N-A 1 / \\
V R N-D 4 \\
\text { exon-3-5 }\end{array}$} & Ex4C.sph & 592 \\
\hline \multirow[t]{3}{*}{ Ex4AR245m3 } & \multirow[t]{3}{*}{ gaccagttcgaataccgaagaa } & & & & Ex4C.f & 588 \\
\hline & & & & & Ex4C.m/ Ex4T & 591 \\
\hline & & & & & Ex4C.s & 590 \\
\hline Ex4outV4F2m3 & cctgtcccacccaaagttacta & & & & Ex4C.sph & 583 \\
\hline Ex4AR245m3 & gaccagttcgaataccgaagaa & [201 & 57 & $V R N-A 1$ & Ex4C.f & 579 \\
\hline & & {$[20]$} & 57 & exon-3-5 & Ex4C.m/ Ex4T & 582 \\
\hline & & & & & Ex4C.s & 581 \\
\hline Ex7Fm4 & caaccttgcataccttcttc & This study & $57-60$ & $V R N-A 1$ & Fx7C & 319 \\
\hline Ex7/C/R2m4 & tttgatcttgctgcgacgc & & $5 /-60$ & exon-6-7 & $\mathrm{Ex} / \mathrm{C}$ & 319 \\
\hline Ex7Fm4 & caaccttgcataccttcttc & This study & $56-58$ & $V R N-A 1$ & Ex7T & 332 \\
\hline $\mathrm{Ex} 7 / \mathrm{T} / \mathrm{R} 2 \mathrm{~m} 3$ & ctaactccgactctttgatcttgctgcgcgac & & & exon-6-7 & & \\
\hline Ex4F2m3 & tgttccttcctgtcccaacc & This study & 57 & $V R N-A 1$ & $\operatorname{Ex} 4(\mathrm{C} / \mathrm{T}) / 7 \mathrm{C}$ & 872 \\
\hline Ex7/C/R2m4 & tttgatcttgctgcgacgc & & & exon-3-7 & Ex4C.f/7C & 870 \\
\hline $\mathrm{Ex} 4 / \mathrm{F} 2 \mathrm{~m} 3$ & tgttccttcctgtcccaacc & This study & 56 & VRN-A1 & $\operatorname{Ex} 4(\mathrm{C} / \mathrm{T}) / 7 \mathrm{~T}$ & 885 \\
\hline Ex7/T/R2m3 & ctaactccgactctttgatcttgctgcgcgac & & & exon-3-7 & 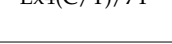 & 000 \\
\hline Ex7Fm4 & caaccttgcataccttcttc & This study & 57 & VRN-A1 & $\operatorname{Ex7}(\mathrm{C} / \mathrm{T})$ & 570 \\
\hline Ex7R & tggatgaatgctgcacaacc & & & exon-6-7 & & \\
\hline
\end{tabular}

\subsection{Polyacrylamide and Agarose Gel Electrophoresis}

PCR fragments were separated on 6.6-8\% non-denaturing polyacrylamide (PAA) gels (mono/bis-acrylamide ratio 82:1) in $1.38 \times$ TBE buffer (123 mM ionic strength), at low temperature $\left(8-15^{\circ} \mathrm{C}\right.$ ), under $8-10 \mathrm{~V} / \mathrm{cm}$. Agarose gel electrophoresis was performed using $1.5 \%$ agarose in $1 \times$ TBE buffer, under $6.6 \mathrm{~V} / \mathrm{cm}$. Visualization of PCR fragments in PAA and agarose gels was conducted using ethidium bromide under UV light using the "Gel Doc XR" (Bio-Rad Laboratories, Inc., Hercules, CA, USA) system. 


\subsection{Design of Allele-Specific Primers for the VRN-A1 Exon 7 Haplotypes}

For the identification of the $V R N-A 1$ exon 7 haplotypes, gene-specific forward and allele-specific (AS) reverse primers were designed (Figure 1). In the forward primer Ex7Fm4, SNPs distinguishing DNA sequences of the VRN1 gene from the A, B and D genomes were localized in positions 2, 3 and 5 bp from the 3' end. An artificial mismatch was added in position 4 . This design completely precludes the amplification of sequences from $V r n-B 1$ and $V r n-D 1$ genes. In the AS reverse primers Ex7/T/R2m3 and Ex7/C/R2m4, the SNP distinguishing the $V R N-A 1$ exon 7 haplotypes was localized in the second position from the 3' end. Furthermore, for the additional destabilization of the DNA double helix, an artificial mismatch was incorporated in the neighboring third position of Ex7/T/R2m3 and fourth position of Ex7/C/R2m4. A non-complementary nucleotide in this position was selected based on adjacent nucleotides as well as on the effect of the mismatch on the stability of the DNA helix [24]. It is assumed that a mismatch at the second position initiates the untwisting of the DNA double helix, overlapping three nucleotides (1-3, from 3' end) which prevents the elongation of DNA by Taq-polymerase. If a mismatch in the second position is absent, a mismatch at the third position (from 3' end) has no significant effect on primer annealing [24].

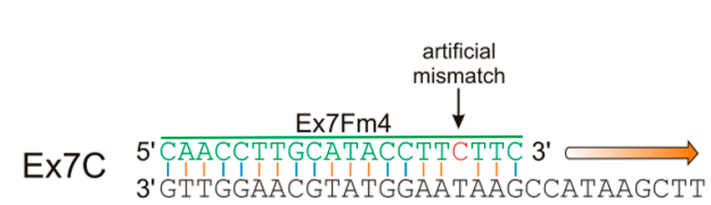

Ex7T

3' GTTGGAACGTATGGAATAAGCCATAAGCTT $\frac{5^{\prime} \text { CAACCTTGCATACCTTCTTC }}{\text { Ex7Fm4 }}$

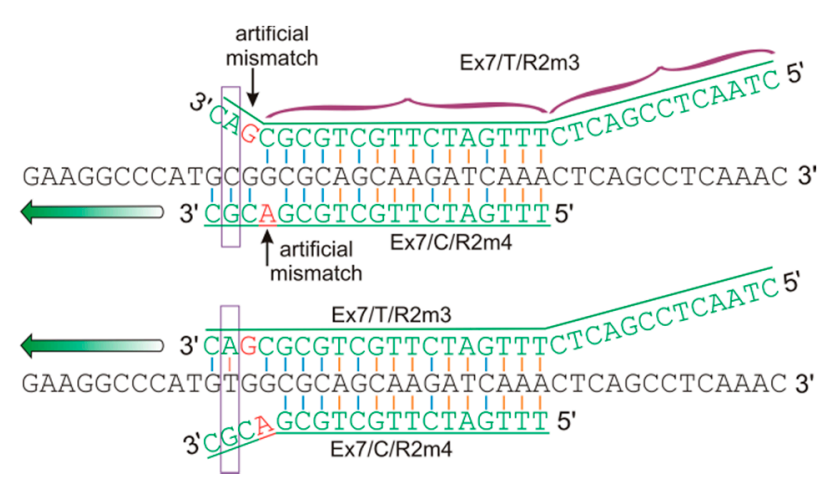

Figure 1. Design of the allele-specific primers Ex7/C/R2m4 and Ex7Fm4-Ex7/T/R2m3 for discrimination of the $V R N-A 1$ exon 7 haplotypes.

Incorporation of a mismatch in the fourth position (from 3' end) of the Ex7/C/R2m4 primer was needed to avoid primer dimmers in multiplex PCR. Furthermore, in order to provide multiplex PCR using both AS primers to VRN-A1 exon 7, the 13 bp non-complement linker was included at the $5^{\prime}$ end of the Ex7/T/R2m3 primer. Multiplex PCR was optimized with the common forward primer Ex7Fm4 that is specific to VRN1 from A genome and two allele-specific reverse primers Ex7/C/R2m4 and Ex7/T/R2m3 for the Ex7C and Ex7T haplotypes, respectively. On the other hand, using separate PCRs with primer pairs Ex7Fm4-Ex7/C/R2m4 and Ex7Fm4-Ex7/T/R2m3 is preferable for operative estimation of the $V R N-A 1$ exon 7 haplotype in agarose gels for the short time of $10 \mathrm{~min}$. Furthermore, singleplex PCR allows us to increase the annealing temperature of Ex7/C/R2m4, thus avoiding false positive results through non-specific amplification. However, it should be noted that during multiplex PCR we did not observe non-specific amplification of alternative alleles even with a decrease in annealing temperature to $54^{\circ} \mathrm{C}$ (Figure 2). All components of the PCR mixture are critical in allele-specific PCR. Across time, the Ex7/T/R2m3 primer showed more stable results than Ex7/C/R2m4. In particular, PCR with Ex7Fm4-Ex7/C/R2m4 can produce non-specific amplicons, which can be distinguished from the target PCR fragments only in PAA gels. This can lead to false positive results during analysis in agarose gels. Thus, PCR with the primer pair Ex7Fm4-Ex7/T/R2m3 should be used for the operative estimation of the VRN-A1 exon 7 haplotype, rather than with the use of Ex7Fm4-Ex7/C/R2m4. 


\subsection{Sequencing of PCR Fragments}

PCR fragments were precipitated with 3 volumes of $96 \%$ ethanol, using linear polyacrylamide $(15 \mu \mathrm{g} / \mathrm{mL})$ as the carrier. If amplification resulted in several target PCR fragments, the desired amplicons were excised from agarose or PAA gels and purified on silica spin columns according to the manufacturer's protocol. Sanger sequencing was carried out with the use of the BigDye Terminator v3.1 sequencing kit, with subsequent analysis on an ABI 3130xl Genetic Analyzer (SB RAS Genomics Core Facility). All sequences reported in this paper were deposited in GenBank under the accession numbers: KU695546 (T. spelta, UA0300246, VRN-A1 Ex4C.f/7C haplotype), KU695547 (T. sphaerococcum, PI 42013, VRN-A1 and VRN-D4 Ex4C.s/7C haplotype), KU695548 (T. aestivum, TRI 828, VRN-A1 Ex4C.m/7T haplotype), MH715939 (T. dicoccoides, IG 113302, VRN-A1 Ex4C.m/7T haplotype), MH715940 (T. dicoccoides, IG 109085, VRN-A1 Ex4C.s/7T haplotype), MH715941 (T. dicoccoides, IG 115808, VRN-A1 Ex4C.s/7T haplotype), MH715942 (T. dicoccoides, IG 46480, VRN-A1 Ex4C.s/7T haplotype), MH715943 (T. aestivum, TRI 3111, VRN-A1 Ex4C.m/7T haplotype), MH715944 (T. compactum, PI 262666, Vrn-A1j Ex4C.m/4T/7T haplotype) and MH715945 (T. sphaerococcum, PI 190982, VRN-A1 Ex4C.m/7T haplotype, VRN-D4 Ex4C.s/7C haplotype).

\section{Results}

\subsection{VRN-A1 exon 7 Polymorphism in Polyploid Wheat}

Allele-specific PCR for the identification of the $V R N-A 1$ exon 7 haplotype was carried out in singleplex as well as in multiplex formats. Using a designed primer set, $319 \mathrm{bp}$ PCR fragments detect the haplotype of the wild type (Ex7C), while $332 \mathrm{bp}$ fragments indicate the mutant exon 7 of $V R N-A 1$ (haplotype Ex7T). The $13 \mathrm{bp}$ difference between the amplicons, which is determined by the different length of allele-specific primers (19 and $32 \mathrm{bp}$ for Ex7/C/R2m4 and Ex7/T/R2m3, respectively), clearly visible in PAA gels, allows the identification of the $V R N-A 1$ exon 7 alternative haplotypes in heterogenous samples during a single reaction (Figure 2).
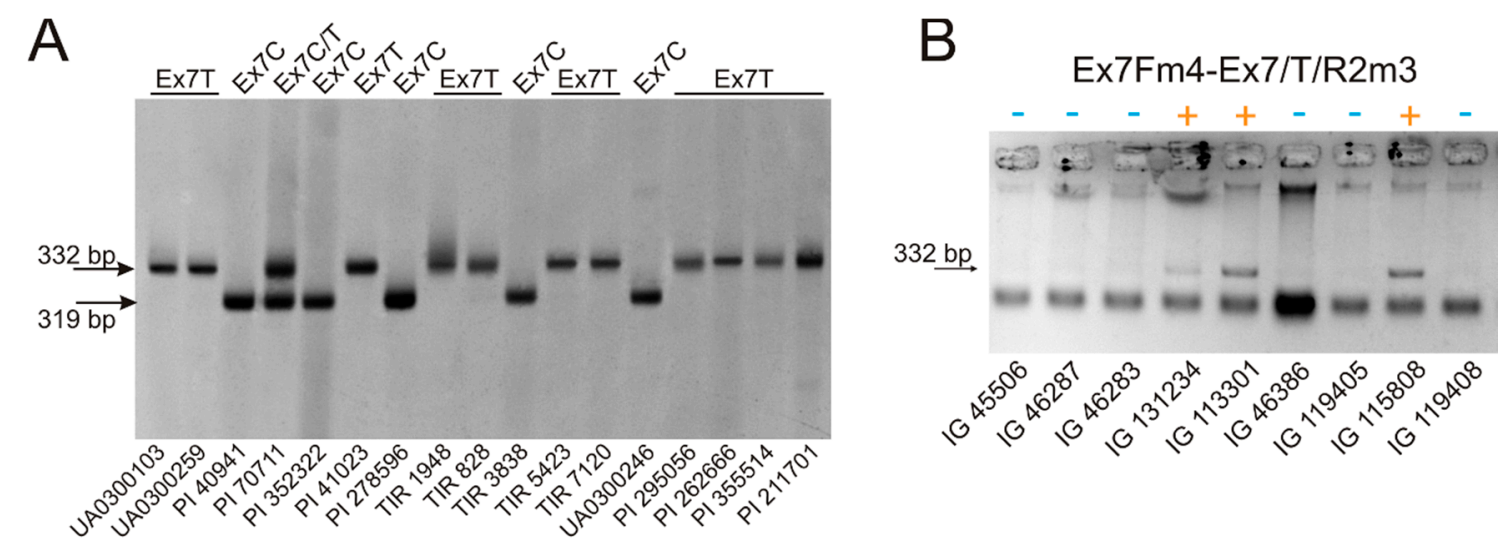

Figure 2. Discrimination of the $V R N-A 1$ exon 7 haplotypes during AS PCR assay. (A) Multiplex PCR with primers Ex7Fm4, Ex7/C/R2m4 and Ex7Fm4-Ex7/T/R2m3. Electrophoresis was carried out in 7\% PAA gel at $10{ }^{\circ} \mathrm{C}$, under $10 \mathrm{~V} / \mathrm{cm}$. The primer annealing temperature was $54{ }^{\circ} \mathrm{C}$; (B) Detection of the Ex7T haplotype was in $1.5 \%$ agarose gel under $6.6 \mathrm{~V} / \mathrm{cm}$ for $10 \mathrm{~min}$. PCR was performed using the Ex7Fm4-Ex7/T/R2m3 primer pair, with an annealing temperature of $57^{\circ} \mathrm{C}$.

Analysis of the $V R N-A 1$ exon 7 in 190 samples of polyploid wheat revealed Ex7C and Ex7T haplotypes in both hexaploid and tetraploid wheat species. In particular Ex7T was detected in a few accessions of $T$. dicoccoides, but no other tetraploid wheat species. In contrast, this haplotype was frequent in hexaploid wheat and was identified in all five species analyzed here. Almost all $V R N-A 1$ alleles carrying mutations within the promoter region and intron- 1 are characterized by the intact 
exon 7, Ex7C haplotype (Table 2), with the exception of the Vrn-A1c (type IL369) and Vrn-A1j alleles, previously identified in hexaploid wheat ([23], GenBank: KU738894) and were not found in tetraploid species ([12,25,26], here).

Table 2. The $V R N-A 1$ exon 4 and exon 7 haplotypes in the different $V R N-A 1$ alleles.

\begin{tabular}{|c|c|}
\hline Allele & $V R N-A 1$ exon 4 and exon 7 Haplotype \\
\hline \multirow[t]{7}{*}{ vrn-A1 } & Ex4C.s/7C \\
\hline & Ex4C.f/7C \\
\hline & Ex4C.s/7T \\
\hline & Ex4C.m/7T \\
\hline & Ex4C.sph/4T/7T \\
\hline & Ex4C.m/4T/7T \\
\hline & Ex4T/7T \\
\hline Vrn-A1a.1-2 & Ex4C.f/7C \\
\hline Vrn-A1b.1-7 & Ex4C.s/7C \\
\hline Vrn-A1e & Ex4C.f/7C \\
\hline Vrn-A1i & Ex4C.f/7C \\
\hline Vrn-A1j (GenBank: KU738894) & Ex4C.m/4T/7T \\
\hline Vrn-A1k (GenBank: KX874608) & Ex4C.s/7C \\
\hline Vrn-A1c (Langdon) & Ex4C.s/7C \\
\hline Vrn-A1c (IL369) & Ex4C.m/4T/7T \\
\hline
\end{tabular}

Samples of T. sphaerococcum (PI 191301, PI 40941, PI 42013, PI 277141, PI 168685) carrying the $V R N-D 4$ gene and the Ex4C haplotype of $V R N-A 1$ exon 4 [20] were characterized by the intact exon 7 of $V R N-A 1$, confirming the Ex7C haplotype of $V R N-D 4$. At the same time, in five accessions of T. sphaerococcum with $V R N-D 4$ (PI 190982, PI 277142, PI 278650, PI 70711, PI 324492) both alternative haplotypes of $V R N-A 1$ exon 7 were detected. However, the exclusion of the VRN-D4 haplotypes during multiplex AS-PCR with the Ex4outV4F2m3 forward primer, previously designed to avoid the amplification of $V R N-D 4$ [20], indicated the Ex7T haplotype for the $V R N-A 1$ gene in these accessions of T. sphaerococcum.

Among samples homozygous for the recessive $v r n$ - $A 1$ allele and without $V R N-D 4$, no genotypes carrying both alternative haplotypes of exon 7 simultaneously were detected among all polyploid wheat species analyzed here. This assumes that in accessions carrying several copies of the VRN-A1 gene, all copies have the same haplotype of exon 7.

\subsection{Combinations of the VRN-A1 Exon 4 and Exon 7 Haplotypes}

The results for the accessions investigated in the present study were added to the analysis of almost 90 publicly available $V R N-A 1$ DNA sequences of diploid and polyploid wheat species. To denote the $V R N-A 1$ exon 4 haplotype, the expanded nomenclature of the $V R N-A 1$ haplotypes was also used [20]. With this nomenclature, mutations within $V R N-A 1$ intron-4 distinguish Ex4C.s, Ex4C.m, Ex4C.f and Ex4C.sph haplotypes (Figure 3). The first three haplotypes were named in accordance with the migration of their corresponding PCR fragments through PAA gel (s: slow, m: middle, f: fast). Ex4C.sph was detected exclusively in T. sphaerococcum. The Ex4T haplotype has the same VRN-A1 intron-4 DNA sequence as the Ex4C.m haplotype.

All dominant $V R N-A 1$ alleles originating in diploid and tetraploid wheat had either Ex4C.s/7C or Ex4C.f/7C haplotype (wild type). Ex4C.f/7C was abundant in T. dicoccoides and T. dicoccum. However, although in T. dicoccoides this haplotype was related to the recessive vrn-A1 allele, in T. dicoccum it was due to the Vrn-A1e and Vrn-A1a.1 alleles, rather than vrn-A1. The Vrn-A1c (IL369) and Vrn-A1j alleles, identified in hexaploid wheat, were characterized by the Ex4C.m/4T/7T haplotype (Table 2, Figure 3D).

Accessions of hexaploid wheat carrying both $V R N-A 1$ exon 4 haplotypes simultaneously (Ex4C, Ex4T), i.e., containing at least two copies of $V R N-A 1$ per haploid genome, had a mutant 
exon 7 of this gene. Using the expanded nomenclature of the $V R N-A 1$ haplotypes, this haplotype is Ex4C.m/7T. Among hexaploid wheat with the Ex4C haplotype alone, the Ex4C.m/7T haplotype was detected in cultivars of T. aestivum from Western Europe, where it was distributed with the haplotype Ex4C.s/7C. One accession of T. spelta and T. aestivum carried the Ex4C.f/7C haplotype alone. Furthermore, in an accession of T. aestivum (TRI 290) the Ex4T/7T haplotype alone was revealed (Table 3).
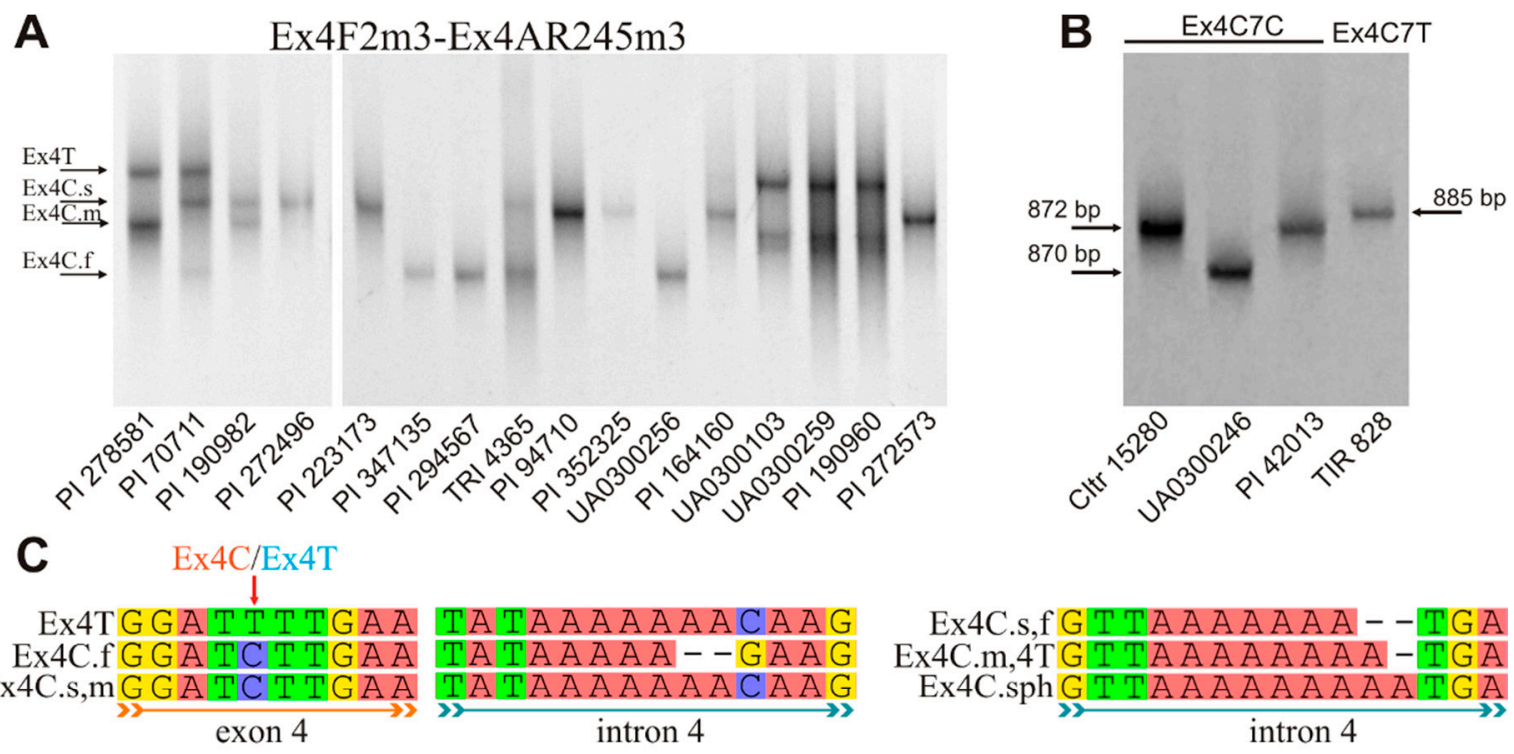

D

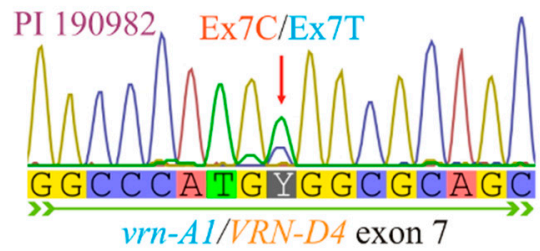

Ex4C.s,fGTTAAAAAAA - -TGA Ex4C.m,4TG T TA A A A A A A - TGA

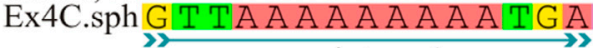
intron 4

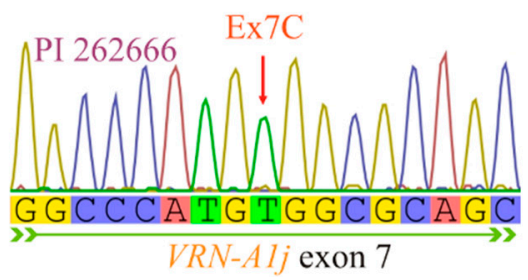

Figure 3. Identification of the $V R N-A 1$ exon 4 and exon 7 haplotypes. (A) Separation of PCR products with primer pair Ex4F2m3-Ex4AR245m 3 in $8 \%$ PAA gel at $10^{\circ} \mathrm{C}$, under $8.75 \mathrm{~V} / \mathrm{cm}$. Accession TRI 4365 is heterozygous at Vrn-A1a.1/vrn-A1; (B) PCR with primer pairs Ex4F2m3-Ex7/C/R2m4 and Ex4F2m3-Ex7/T/R2m3 for the identification of the $V R N-A 1$ exon 4 haplotype in copies of this gene with the intact (Ex7C) and mutant (Ex7T) exon 7, respectively; (C) Polymorphism of the VRN-A1 exon 4 and intron 4 , which distinguishes different haplotypes of this gene (in accordance with the expanded nomenclature); (D) Chromatograms of the $V R N-A 1$ exon 7 sequencing. The double fluorescence peak (Ex7T/Ex7C) for the accession of T. sphaerococcum PI 190982 carrying VRN-D4 and a single peak for the accession of T. compactum PI 262666 with $V R N-A 1 j$ are indicated.

Overall, the mutant type of exon 4 (Ex4T) was found to be associated with the mutant type of exon 7 (Ex7T) of the VRN-A1 gene (haplotype Ex4T7T). Similarly, the wild type of exon 7 (Ex7C) is associated with the wild type exon $4(\mathrm{Ex} 4 \mathrm{C})$, since no variants with the haplotype Ex4T7C were detected. Using the expanded subset of $V R N-A 1$ haplotypes, an association was found between the Ex4C.m haplotype and the mutant exon 7 of $V R N-A 1$. Sequence analysis of PCR fragments has also shown that the Ex4C.m/7T haplotype carries the deletion of " $\mathrm{A}$ " within intron 6, which agrees with previous results [17].

The seventh exon of $V R N-A 1$ in accessions of T. sphaerococcum with the Ex4C haplotype alone (PI 191301, PI 40941, PI 42013, PI 277141, PI 168685, CItr 17737) is represented by the wild type (Ex7C), indicating that the VRN-D4 gene is also characterized by haplotype Ex4C7C. However, in two accessions of T. sphaerococcum with haplotype Ex4C (PI 190982 and PI 278650), both the wild and mutant type of exon 7 were detected simultaneously (Figure 3D). Exclusion of the haplotype Ex4C7C of 
$V R N-D 4$ leads to the conclusion that the haplotype of the $V R N-A 1$ gene in these samples corresponds to Ex4C7T. This was also confirmed using the $V R N-A 1$ specific Ex4outV4F2m3 forward primer in combination with allele-specific primers for the identification of the $V R N-A 1$ exon 7 haplotypes.

Furthermore, three accessions of T. sphaerococcum (PI 277142, PI 70711 and PI 324492) were identified containing alternative haplotypes for both the fourth and seventh exons of $V R N-A 1$ simultaneously. In order to determine the combination of the $V R N-A 1$ exon 4 and exon 7 haplotypes for each copy of this gene, these samples were amplified by the primer combinations Ex4F2m3-Ex7TR2m3 and Ex4F2m3-Ex7CR2m4. In this PCR, the forward primer is genome-specific (used to identify the exon 4 haplotype), while the reverse primers are allele-specific to the exon 7 haplotypes. Thus, the amplification region encompasses the nucleotide sequences of polymorphic sites within the fourth and seventh exons simultaneously. As a result, the observed difference in the migration rate of PCR fragments, with a differing curvature of the DNA molecules, allows one to determine the exon 4 haplotype for each copy of the $V R N-A 1$ gene with a specific haplotype of exon 7 . Segregation of the exon 4 haplotypes was observed only for amplicons with the mutant type of exon 7 , confirming that the Ex7C haplotype in these accessions of T. sphaerococcum belongs to the VRN-D4 gene. Finally, no PCR fragments were obtained using the $V R N-A 1$-specific forward primer in combination with the AS reverse primer to Ex7C.

Table 3. Distribution of the $V R N-A 1$ exon 4 and exon 7 haplotypes in polyploid wheat with the recessive vrn-A1 allele *.

\begin{tabular}{|c|c|c|}
\hline VRN-A1 Haplotype & Tetraploid Wheat & Hexaploid Wheat \\
\hline Ex4C.s/7C & $\begin{array}{l}\text { T. dicoccoides (PI 352325, PI 428018, } \\
\text { IG 46287) } \\
\text { T. dicoccum (TRI 28027, TRI 16880) } \\
\text { T. carthlicum (PI 115817, PI 190949, } \\
\text { PI 272521, PI 283887, PI 352279, } \\
\text { PI 532505, PI 532512) } \\
\text { T. turgidum (PI 264991) }\end{array}$ & $\begin{array}{l}\text { T. aestivum (TRI 867, TRI 4560, TRI 3838, } \\
\text { TRI 9533, TRI 7129) } \\
\text { T. sphaerococcum (PI 191301, PI 40941, PI 42013, } \\
\text { PI 277141, PI 168685, CItr 17737) }\end{array}$ \\
\hline Ex4C.f/7C & $\begin{array}{l}\text { T. dicoccoides (PI 352322, } \\
\text { UA0300256, IG 46386, IG 46527, } \\
\text { IG 46353) } \\
\text { T. dicoccum (K-21416) }\end{array}$ & $\begin{array}{l}\text { T. aestivum (TRI 3088) } \\
\text { T. spelta (UA0300246) }\end{array}$ \\
\hline Ex4C.s/7T & $\begin{array}{l}\text { T. dicoccoides (IG 109085, IG 115808, } \\
\text { IG 46480) }\end{array}$ & \\
\hline Ex4C.m/7T & $\begin{array}{l}\text { T. dicoccoides (IG 131234, IG 113301, } \\
\text { IG 131233, IG 109088, IG 131232, } \\
\text { IG 113302) }\end{array}$ & $\begin{array}{l}\text { T. aestivum (TRI 828, TRI 834, TRI 4917, } \\
\text { TRI 1948, TRI 3111, TRI 4925) } \\
\text { T. sphaerococcum (PI 190982, PI 278650) }\end{array}$ \\
\hline Ex4T/7T & & T. aestivum (TRI 290) \\
\hline Ex4C.m/4T//7T & & $\begin{array}{l}\text { T. aestivum (TRI 1712, TRI 3534, TRI 4078, } \\
\text { TRI 4739, TRI 5395, TRI 950, TRI 2979, } \\
\text { TRI 3891, TRI 5310, TRI 5394, TRI 5423, } \\
\text { TRI 7120, TRI 8459, TRI 11031) } \\
\text { T. compactum (PI 186391, PI 157920, PI 129523, } \\
\text { PI 211701, PI 278541, PI 262666, PI 278581, } \\
\text { PI 352306, PI 41023) } \\
\text { T. spelta (UA0300103, UA0300259, PI 190960, } \\
\text { PI 225271, PI 286048, PI 295056, PI 347850) } \\
\text { T. macha (PI 272554, PI 352466, PI 355511, } \\
\text { PI 355514, PI 428146, PI 428179, PI 542466, } \\
\text { PI 572905) }\end{array}$ \\
\hline Ex4C.sph/4T/7T & & $\begin{array}{l}\text { T. sphaerococcum (PI 277142, PI 70711, } \\
\text { PI 324492) }\end{array}$ \\
\hline
\end{tabular}

* Accessions with the recessive vrn-A1 allele were not present in T. durum and T. polonicum. 
For those remaining among samples with the intact exon 4 of $V R N-A 1$ (Ex4C), genotypes carrying both alternative $V R N-A 1$ exon 7 haplotypes simultaneously were not found. This assumes the absence of variants with two or more copies of the $V R N-A 1$ gene per haploid genome, which are characterized by the Ex4C7C and Ex4C7T haplotypes.

\section{Discussion}

Previously, the $V R N-A 1$ exon 7 polymorphism was analyzed in a small subset of $T$. aestivum varieties. In the present study, the number of hexaploid wheat species was increased to five. Furthermore, the $V R N-A 1$ exon 7 was analyzed in six tetraploid wheat species for the first time. As a result, all the species of polyploid wheat most often involved in breeding, as well as known $V R N-A 1$ alleles occurring in them were encompassed.

In previous studies, DNA-markers, based on the restriction cleavage of PCR fragments (CAPS) and (Kompetitive allele specific PCR) KASP assay were proposed for the identification of the VRN-A1 exon 7 haplotype [15-18]. These markers are dominant, and they do not allow us to analyze the $V R N-A 1$ exon 4 and exon 7 simultaneously, nor do they do allow us to exclude $V R N-D 4$ or genotype heterozygotes at the $V R N-A 1$ exon 7 . Conversely, allele-specific PCR markers allow us to avoid the disadvantages described above. In the design of AS primers for $V R N-A 1$ exon 7 haplotypes, we worked on the same principles as in our previous study of $V R N-A 1$ exon 4 polymorphism [20]. It should be noted that during PCR with the proposed AS primers for the $V R N-A 1$ exon 4 and exon 7 haplotypes, false positive results through the amplification of alternative alleles, were absent. For this reason, it is assumed that the localization of the SNP in the second position (from the $3^{\prime}$ end) and the incorporation of an artificial mismatch in the third position is an effective approach in the design of allele-specific primers for SNP genotyping.

It has long been assumed that the mutations $\mathrm{Leu}^{117} / \mathrm{Phe}^{117}(\mathrm{Ex} 4 \mathrm{C} / \mathrm{Ex} 4 \mathrm{~T})$ and $\mathrm{Ala}^{180} / \mathrm{Val}^{180}$ (Ex7C/Ex7T) are associated [27]. However, it should be noted that the multicopy nature of $V R N-A 1$ and, hence, the presence in the genome of several copies of this gene with alternative haplotypes of exon 4 and exon 7 in different combinations, were not taken into account in these studies. Later it was shown that haplotype Ex4C can be represented in different combinations with alternative haplotypes of exon $7[16,18]$. In particular, the combination Ex4C/4T/7T was identified in accessions carrying 2-3 copies of $V R N-A 1$ [18]. For this reason, it has been assumed that the haplotype of $V R N-A 1$ exon 7 cannot be identified based on the known haplotype of exon 4 of this gene and vice versa [16]. In the present study it was determined that the wild type of exon 7 (Ex7C) and mutant type of exon 4 (Ex4T) are associated with the analogous haplotypes of exon 4 (Ex4C) and exon 7 (Ex7T), respectively. Thus, the Ex7T and Ex4C haplotypes can be predicted based on the presence of the Ex4T and Ex7C haplotypes, respectively, in the given copy of the VRN-A1 gene.

In previous studies, it was noted that the Ex7C haplotype predominates in spring varieties, whereas the Ex7T haplotype is mainly detected in winter varieties of T. aestivum $[13,17,27]$. There was a suggestion that the Ex7C haplotype is associated with the spring growth habit, while the Ex7T haplotype correlates with a strong vernalization requirement and winter growth habit $[13,17,27]$. In this study, it was found that almost all known dominant $V R N-A 1$ alleles have the Ex7C haplotype, while the vast majority of hexaploid wheat accessions with the recessive vrn-A1 allele are characterized by the haplotype Ex7T. In particular, the presence of two or more copies of the VRN-A1 gene (as found, according to the latest data, in 95\% of winter varieties of T. aestivum [19]), is associated with the Ex7T haplotype, which is the scientific explanation for previous observations. Furthermore, since the wild type of the VRN-A1 exons 4 and 7 (Ex4C/7C haplotype) was found to be characteristic of the VRN-D4 gene as well as the vast majority of known dominant $V R N-A 1$ alleles, the need to identify haplotypes of $V R N-A 1$ exons 4 and 7 is only relevant for samples carrying the recessive vrn-A1 allele, and the need to identify the $V R N-D 4$ gene is relevant only for hexaploid wheat carrying the Ex4C/7C haplotype.

The associations between the $V R N-A 1$ exon 4 and exon 7 haplotypes revealed here in different wheat species with different $V R N-A 1$ alleles make it possible to assume the divergence of combinations 
of these haplotypes during wheat evolution, as represented in Figure 4. According to the inferred phylogenetic relationships between the alternative haplotypes of $V R N-A 1$ exons 4 and 7 , the divergence of the seventh exon occurred earlier than the fourth exon. This assumes that the second and third copies of $V R N-A 1$ with the Ex4T/7T haplotype diverged sequentially in the phylogenetic lineage of the Ex4C.m/7T haplotype. For this reason, it is assumed that the presence of multiple copies of $V R N-A 1$ in hexaploid wheat is associated with the Ex4T mutant type of exon 4 and the Ex7T mutant type of exon 7, resulting in the combinations of Ex4C.m/7T, Ex4T/7T and Ex4C.m/7T, Ex4T/7T, Ex4T/7T for two and three copies of the $V R N-A 1$ gene, respectively. Thus, the $V R N-A 1$ exon 4 polymorphism, observed exclusively in hexaploid wheat with at least two copies of this gene per haploid in the genome [20], is associated with the mutant type of exon 7. Although accession TRI 290 carries the Ex4T/7T haplotype, we cannot rule out that this accession is mutant.

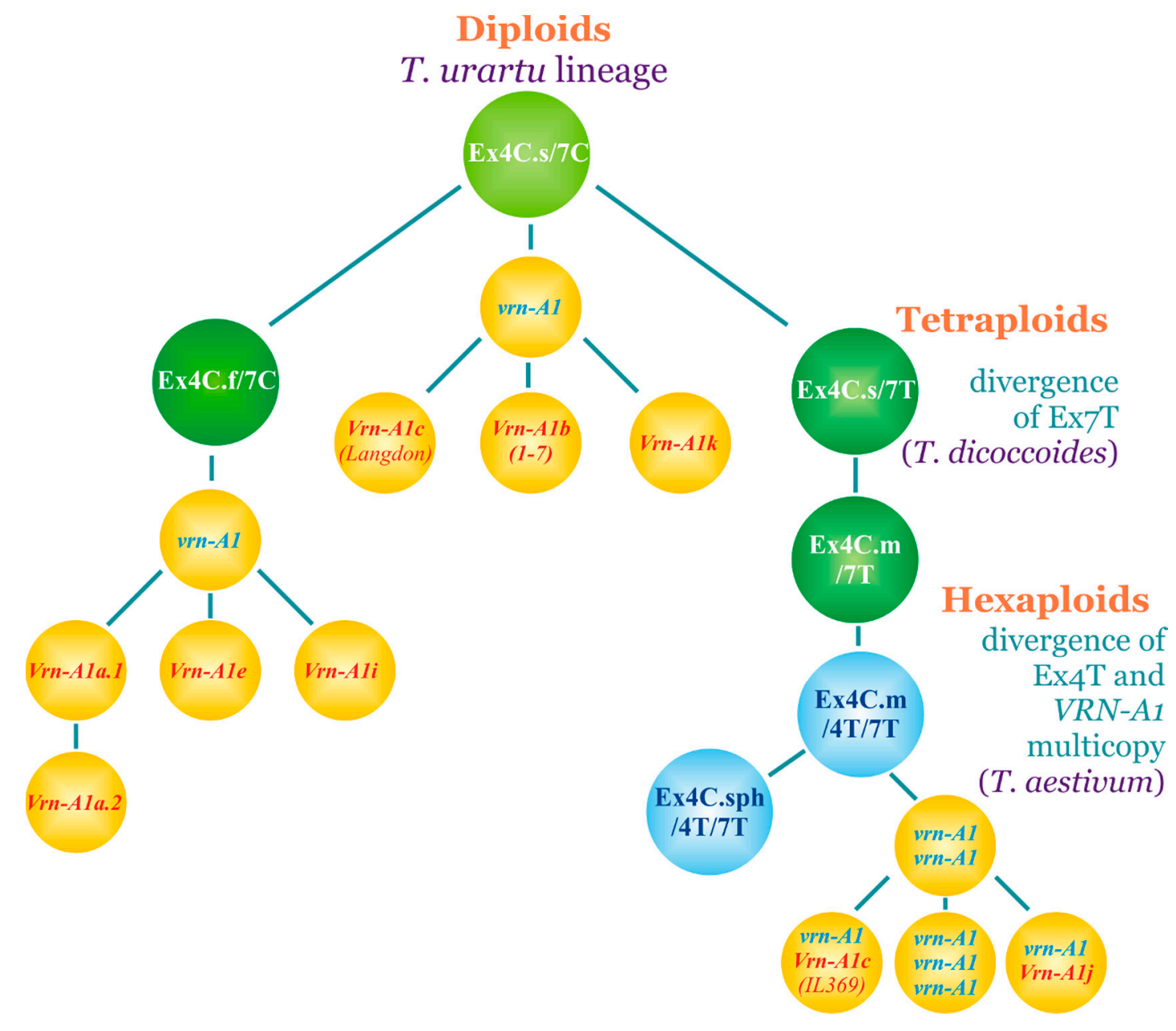

Figure 4. Divergence of the $V R N-A 1$ exon 4 and exon 7 haplotypes and $V R N-A 1$ alleles. Only the $V R N-A 1$ alleles identified in the present study or alleles with publicly available DNA sequences with overlapping exons 4 and 7 are represented.

Surprising results were obtained for spelt wheat species (T. spelta and T. macha). Despite the fact that Asian and European spelt are polyphyletic, it is assumed that they were derived from hybridization of hulled emmer T. dicoccum with free-threshing hexaploid wheat T. aestivum $[28,29]$. For this reason, we expected a split at the $V R N-A 1$ exon 4 and exon 7 haplotypes in some accessions of $T$. spelta and T. macha, along with accessions carrying one haplotype of this gene. However, all analyzed accessions of spelt wheat with recessive vrn-A1 that split at the exon 4 haplotypes (Ex4C.m and Ex4T copies of $V R N-A 1)$ are characterized by the Ex7T haplotype alone. This is especially interesting since the mutant 
$V R N-A 1$ exon 7, as well as Ex4C.m haplotype have not been identified in T. dicoccum. Although it seems unlikely we cannot rule out that all of these accessions are homozygous at $V R N-A 1$. Other accessions of T. spelta and T. macha carried Vrn-A1b.2 (nine accessions), vrn-A1b.3 (2 accessions) and for one accession of T. spelta the Vrn-A1b.1 was identified. In our previous study, with the exception of spelt wheat, the Vrn-A1b.2 allele was abundant in T. dicoccoides and T. dicoccum [12], but not in the other tetraploid and hexaploid wheat species analyzed here. This fact may provide evidence for the emergence of spelt wheat from the hybridization of the $\mathrm{D}$ genome donor (Ae. tauschii or T. aestivum) with T. dicoccum. Similarly, with the exclusion of one accession of T. spelta with the Ex4C.f/7C haplotype (also observed in $T$. dicoccum) all accessions of spelt wheat with recessive $v r n-A 1$ had the Ex4C.m/4T/7T haplotype. While polymorphism of $V R N-A 1$ exon 4 and the $V R N-A 1$ multicopy are the latter evolutionary events, the more ancient Ex4C.s/7C, Ex4C.s/7T and Ex4C.m/7T haplotypes alone were found in cultivars of T. aestivum, but not in T. spelta and T. macha, which assumes that accessions of spelt wheat with the Ex4C.m/4T/7T haplotype likely originated from T. aestivum. In any case, the obvious polyphyletic emergence of polyploid wheat of both T. aestivum and spelt wheat, make it difficult to interpret the obtained results.

Among the VRN-A1 alleles, the Ex7T haplotype was detected only in Vrn-A1c (IL369) and Vrn-A1j. The coding sequences of these alleles are also characterized by the exon 4 polymorphism, which is not typical for any known $V R N-A 1$ alleles. Furthermore, in contrast to the other $V R N-A 1$ alleles, $V r n-A 1 c$ (IL369) and Vrn-A1j are associated with at least one recessive $v r n-A 1$ copy ([18], this study). Thus, accessions carrying these alleles include at least two copies (mutant and intact) of VRN-A1 per haploid genome. The results of this investigation of the $V R N-A 1$ exon 4 and exon 7 haplotype distribution in different species of polyploid wheat, analysis of combinations of these haplotypes, their phylogenetic relationships and associations with the different alleles and the number of copies of this gene allows us to conclude that the further emergence of the new $V R N-A 1$ alleles in hexaploid wheat have a high probability (in most cases) of being associated with the presence of several copies of $V R N-A 1$, a part of which remain intact (recessive vrn- $A 1$ allele), and the coding sequence will carry C->T transitions within exon 4 and/or exon 7 of $V R N-A 1$. This assumption is consistent with the results of the analysis of the Vrn-A1c (IL369) and Vrn-A1j alleles, which contrast with the emergence of the other $V R N-A 1$ alleles in hexaploid wheat, and possesses the features described above.

Decreasing genetic variation during the domestication of wheat is due to the two mutually associated evolutionary events of genetic bottleneck and the founder effect [30,31]. Nevertheless, as is known, polyploid wheat compensated for this decrease in genetic diversity, caused by the domestication and polyploidization events, through the genetic variability of its tetraploid wild progenitor [32]. In fact, all combinations of $V R N-A 1$ exon 4 and exon 7 haplotypes observed in wild emmer $T$. dicoccoides were found in bread wheat $T$. aestivum. Furthermore, the genetic variation of hexaploid wheat species has been enriched with novel alleles, such as Vrn-A1c (IL369) (in T. aestivum [23]) and Vrn-A1j (in T. compactum), as well as the new combination of Ex4T/7T and copy number variation of $V R N-A 1$ with different proportions of Ex4C/7T and Ex4T/7T haplotypes. Analysis of the relationships between the $V R N-A 1$ exon 4 and exon 7 haplotypes found no less than two genetic variation bottlenecks during the domestication and evolution of wheat (Figure 4). The first is associated with the divergence of Vrn-A1a.1, Vrn-A1e and Vrn-A1i alleles in the population of tetraploid wheat carrying the Ex4C.f/7C haplotype. It is likely that this population is T. dicoccum, since these alleles were not observed in $T$. dicoccoides $([12,25,26]$, this study). The second event is related to the presence of multiple copies of $V R N-A 1$ in the population of hexaploid wheat with the Ex4C.m/7T haplotype following the divergence of the $V R N-A 1$ Ex4T/7T copy.

\section{Conclusions}

The divergence of the seventh exon of $V R N-A 1$ occurred earlier than the fourth exon, in the wild emmer T. dicoccoides, while polymorphism of the $V R N-A 1$ exon 4 was observed in domesticated, hexaploid wheat. In $V R N-A 1$, the intact exon 7 and mutant exon 4 are associated with analogous types 
of exon 4 and 7, respectively. Accessions of hexaploid wheat containing at least two copies of $V R N-A 1$ with alternative exon 4 haplotypes per haploid genome carry mutant exon 7 of this gene. Hexaploid wheat T. aestivum includes most combinations of the $V R N-A 1$ exon 4 and exon 7 haplotypes found among polyploid wheat. Furthermore, the ratio of the $V R N-A 1$ copies with the alternative exon 4 and exon 7 haplotypes, as well as the number of these copies per genome, increase the total variants of the $V R N-A 1$ genotype significantly. This can contribute to the high adaptability of wheat to a wide range of environmental conditions and, hence, the widespread distribution of this wheat species throughout the world. Thus, we conclude that genetic changes during the domestication of wheat have led to an increase in VRN-A1 diversity at the level of translation, which in turn can facilitate an increase in the adaptive potential of modern hexaploid wheat species to varying environments.

Author Contributions: A.M. carried out the experiments, data analysis and wrote the manuscript. E.A. revised the manuscript, contributed to the discussion, and participated in preparing the manuscript. All authors read and approved the final manuscript.

Funding: This research was funded by the Russian Scientific Foundation grant number 18-74-00080.

Acknowledgments: We are grateful to Carly Schramm for comments and English polishing of the manuscript.

Conflicts of Interest: The authors declare no conflict of interest.

\section{References}

1. Salamini, F.; Ozkan, H.; Brandolini, A.; Schäfer-Pregl, R.; Martin, W. Genetics and geography of wild cereal domestication in the near east. Nat. Rev. Genet. 2002, 3, 429-441. [CrossRef] [PubMed]

2. Kihara, H. Discovery of the DD-analyser, one of the ancestors of Triticum vulgare (Japanese). Agric. Hortic. 1944, 19, 13-14.

3. FAO: FAOSTAT Database. (Crops: Wheat: Area Harvested: 2014). Available online: http://www.fao.org/ faostat/ (accessed on 24 June 2018).

4. Worland, A.J. The influence of flowering time genes on environmental adaptability in European wheats. Euphytica 1996, 89, 49-57. [CrossRef]

5. Snape, J.W.; Butterworth, K.; Whitechurch, E.; Worland, A.J. Waiting for fine times: Genetics of flowering time in wheat. Euphytica 2001, 119, 185-190. [CrossRef]

6. Kamran, A.; Iqbal, M.; Spaner, D. Flowering time in wheat (Triticum aestivum L.): A key factor for global adaptability. Euphytica 2014, 197, 1-26. [CrossRef]

7. Yan, L.; Loukoianov, A.; Tranquilli, G.; Helguera, M.; Fahima, T.; Dubcovsky, J. Positional cloning of the wheat vernalization gene VRN1. Proc. Natl. Acad. Sci. USA 2003, 100, 6263-6268. [CrossRef] [PubMed]

8. Yan, L.; Loukoianov, A.; Blechl, A.; Tranquilli, G.; Ramakrishna, W.; SanMiguel, P.; Bennetzen, J.; Echenique, V.; Dubcovsky, J. The wheat VRN2 gene is a flowering repressor down-regulated by vernalization. Science 2004, 303, 1640-1644. [CrossRef] [PubMed]

9. Yan, L.; Fu, D.; Li, C.; Blechl, A.; Tranquilli, G.; Bonafede, M. The wheat and barley vernalization gene VRN3 is an orthologue of FT. Proc. Natl. Acad. Sci. USA 2006, 103, 19581-19586. [CrossRef] [PubMed]

10. Kippes, N.; Debernardi, J.M.; Vasquez-Gross, H.A.; Akpinar, B.A.; Budak, H.; Kato, K.; Chao, S.; Akhunov, E.; Dubcovsky, J. Identification of the VERNALIZATION 4 gene reveals the origin of spring growth habit in ancient wheats from South Asia. Proc. Natl. Acad. Sci. USA 2015, 112, E5401-E5410. [CrossRef] [PubMed]

11. Muterko, A.F.; Balashova, I.A.; Fayt, V.I.; Sivolap, Y.M. Molecular genetic mechanisms of regulation of growth habit in wheat. Cytol. Genet. 2015, 49, 58-71. [CrossRef]

12. Muterko, A.; Kalendar, R.; Salina, E. Novel alleles of the VERNALIZATION1 genes in wheat are associated with modulation of DNA curvature and flexibility in the promoter region. BMC Plant Biol. 2016, 16, 9. [CrossRef] [PubMed]

13. Chen, Y.; Carver, B.F.; Wang, S.; Zhang, F.; Yan, L. Genetic loci associated with stem elongation and winter dormancy release in wheat. Theor. Appl. Genet. 2009, 118, 881-889. [CrossRef] [PubMed]

14. Eagles, H.A.; Cane, K.; Trevaskis, B. Veery wheats carry an allele of Vrn-A1 that has implications for freezing tolerance in winter wheats. Plant Breed. 2011, 130, 413-418. [CrossRef] 
15. Li, G.; Yu, M.; Fang, T.; Cao, S.; Carver, B.F.; Yan, L. Vernalization requirement duration in winter wheat is controlled by TaVRN-A1 at the protein level. Plant J. 2013, 76, 742-753. [CrossRef] [PubMed]

16. Yan, L.; Li, G.; Yu, M.; Fang, T.; Cao, S.; Carver, B.F. Genetic mechanisms of vernalization requirement duration in winter wheat cultivars. In Advances in Wheat Genetics: From Genome to Field; Ogihara, Y., Takumi, S., Handa, H., Eds.; Springer: Tokyo, Japan, 2015; Volume 13, pp. 117-125.

17. Sherman, J.D.; Yan, L.; Talbert, L.; Dubcovsky, J. A PCR Marker for Growth Habit in Common Wheat Based on Allelic Variation at the VRN-A1 Gene. Crop Sci. 2004, 44, 1832-1838. [CrossRef]

18. Diaz, A.; Zikhali, M.; Turner, A.; Isaac, P.; Laurie, D. Copy number variation affecting the Photoperiod-B1 and Vernalization-A1 genes is associated with altered flowering time in wheat (Triticum aestivum). PLoS ONE 2012. [CrossRef] [PubMed]

19. Würschum, T.; Boeven, P.H.; Langer, S.M.; Longin, C.F.; Leiser, W.L. Multiply to conquer: Copy number variations at Ppd-B1 and Vrn-A1 facilitate global adaptation in wheat. BMC Genet. 2015, 29, 16-96. [CrossRef] [PubMed]

20. Muterko, A.F.; Salina, E.A. Analysis of the VERNALIZATION-A1 exon-4 polymorphism in polyploid wheat. Vavilov J. Genet. Breed. 2017, 21, 323-333. [CrossRef]

21. Masoomi-Aladizgeh, F.; Jabbari, L.; Khayam Nekouei, R.; Aalami, A. A simple and rapid system for DNA and RNA isolation from diverse plants using handmade kit. Protoc. Exch. 2016. [CrossRef]

22. Yan, L.; Helguera, M.; Kato, K.; Fukuyama, S.; Sherman, J.; Dubcovsky, J. Allelic variation at the VRN1 promoter region in polyploid wheat. Theor. Appl. Genet. 2004, 109, 1677-1686. [CrossRef] [PubMed]

23. Fu, D.; Szucs, P.; Yan, L.; Helguera, M.; Skinner, J.S.; von Zitzewitz, J.; Hayes, P.M.; Dubcovsky, J. Large deletions within the first intron in VRN-1 are associated with spring growth habit in barley and wheat. Mol. Genet. Genom. 2005, 273, 54-65. [CrossRef] [PubMed]

24. Liu, J.; Huang, S.; Sun, M.; Liu, S.; Liu, Y.; Wang, W.; Zhang, X.; Wang, H.; Hua, W. An improved allele-specific PCR primer design method for SNP marker analysis and its application. Plant Methods 2012, 8, 34. [CrossRef] [PubMed]

25. Konopatskaia, I.; Vavilova, V.; Kondratenko, E.Y.; Blinov, A.; Goncharov, N.P. VRN1 genes variability in tetraploid wheat species with a spring growth habit. BMC Plant Biol. 2016, 16, 244. [CrossRef] [PubMed]

26. Shcherban, A.B.; Strygina, K.V.; Salina, E.A. VRN-1 gene- associated prerequisites of spring growth habit in wild tetraploid wheat T. dicoccoides and the diploid A genome species. BMC Plant Biol. 2015, 15, 94. [CrossRef] [PubMed]

27. Chen, L.; Wang, S.Q.; Hu, Y.G. Detection of SNPs in the VRN-A1 gene of common wheat (Triticum aestivum L.) by a modified Ecotilling method using agarose gel electrophoresis. Aus. J. Crop Sci. 2011, 5, 321-329.

28. Dvorak, J.; Deal, K.R.; Luo, M.C.; You, F.M.; von Borstel, K.; Dehghani, H. The origin of spelt and free-threshing hexaploid wheat. J. Hered. 2012, 103, 426-441. [CrossRef] [PubMed]

29. Jaaska, V. NADP-dependent aromatic alcohol dehydrogenase in polyploid wheats and their diploid relatives. On the origin and phylogeny of polyploid wheats. Theor. Appl. Genet. 1978, 53, 209-217. [CrossRef] [PubMed]

30. Haudry, A.; Cenci, A.; Ravel, C.; Bataillon, T.; Brunel, D.; Poncet, C.; Hochu, I.; Poirier, S.; Santoni, S.; Glémin, S.; et al. Grinding up wheat: a massive loss of nucleotide diversity since domestication. Mol. Biol. Evol. 2007, 24, 1506-1517. [CrossRef] [PubMed]

31. Feldman, M.; Levy, A.A. Genome evolution due to allopolyploidization in wheat. Genetics 2012, 192, $763-774$. [CrossRef] [PubMed]

32. Dubcovsky, J.; Dvorak, J. Genome plasticity a key factor in the success of polyploid wheat under domestication. Science 2007, 316, 1862-1866. [CrossRef] [PubMed]

(C) 2018 by the authors. Licensee MDPI, Basel, Switzerland. This article is an open access article distributed under the terms and conditions of the Creative Commons Attribution (CC BY) license (http:/ / creativecommons.org/licenses/by/4.0/). 